\title{
Simulated Microbe Removal around Finger Rings Using Different Hand Sanitation Methods
}

\author{
Archana A. Alur ${ }^{1}$, Madhavi J. Rane ${ }^{2}$, James P. Scheetz ${ }^{3}$, Douglas J. Lorenz ${ }^{4}$, Lawrence Gettleman ${ }^{3 *}$ \\ ${ }^{1}$ Ballard High School, Louisville, Kentucky, USA \\ ${ }^{2}$ School of Medicine, University of Louisville, Louisville, Kentucky, USA \\ ${ }^{3}$ School of Dentistry, University of Louisville, Louisville, Kentucky, USA \\ ${ }^{4}$ School of Public Health \& Information Sciences, University of Louisville, Louisville, Kentucky, USA
}

\begin{abstract}
Archana A. Alur, Madhavi J. Rane, James P. Scheetz, Douglas J. Lorenz, Lawrence Gettleman. Simulated Microbe Removal around Finger Rings Using Different Hand Sanitation Methods. International Journal of Oral Science, 1(3): 136-142, 2009
\end{abstract}

\begin{abstract}
Aim It is our opinion that the CDC and the WHO have underestimated cross-contamination under examination gloves in dental clinics while wearing jewelry, such as finger rings. These agencies only "recommend" removing jewelry, and only washing hands for 15 seconds with soap and warm water before donning gloves. This study examined several washing procedures and finger rings using simulated microbes.
\end{abstract}

Methodology A gloved rubber hand manikin was made and fitted with a fresh disposable vinyl glove. Four fingers were fitted with rings or no ring, dusted with simulated microbes, and washed with a scrub brush for 5,15 , and 25 seconds under $20^{\circ} \mathrm{C}$ and $40^{\circ} \mathrm{C}$ water alone, or with liquid hand soap. Light levels (in lux) of fluorescent powder before and after washing were measured and delta scores calculated for changes in light levels, equivalent to effectiveness of hand washing procedures.

A full-factorial, 3-factor analysis of variance (ANOVA) was used to test for differences among levels of the three study factors - time, temperature, and soap use. Tukey's post hoc honestly significant difference (HSD) test was applied to significant factors to examine pair-wise differences between factor levels.

Results It was found that the longer the hands with rings were washed with a scrub brush under flowing water, the more simulated microbes were removed. By 25 seconds, all methods were essentially the same. Simulated microbes were more difficult to remove from the palm compared to the back of the hand. The liquid hand soap used in this study was more effective with warm water than cold. When given a choice of washing with cold water up to 15 seconds, it would be preferable not to use soap to remove simulated microbes. Qualitatively, the outer surface of finger rings were more effectively cleaned than the crevice below the ring, and the ring with a stone setting appeared to accumulate and retain simulated microbes more than other rings.

Conclusion The most effective treatment was washing with warm water and liquid soap. Longer times were more effective. Rings should not be worn under examination gloves due to difficulty cleaning in the crevice under the ring, and the well-known consequences of cross-contamination between the patient and the health care worker.

Keywords dental exam gloves, finger ring jewelry, crosscontamination, hand hygiene, microbes, fluorescent powder, dental health care workers
Document code: A

$$
\text { CLC number: R472.1 }
$$

\section{Introduction}

Microbes are present in and around the finger rings. In spite of hand washing procedures, microbes
Received Aug. 1, 2009; Revision accepted Aug. 24, 2009

will persist. The US Centers for Disease Control \& Prevention (CDC Morbidity \& Mortality Weekly Report, 2002, Guideline for Hand Hygiene in Health Care Settings), and the World Health Orga- 
nization (WHO Guidelines on Hand Hygiene in Health Care, 2005) only make "recommendations" regarding the removal of finger rings and other hand jewelry by health care workers. Two journal publications are cited that examined the effects of jewelry on cross-contamination (pp.30 and pp.7172, respectively). One of them by Salisbury et al. (1997) found that rings do cross-contaminate, but the abovementioned references say the opposite. The other one by Jacobson et al. (1985) found that there were initial differences in bacterial counts between hands with rings and those without, which equalized only after thorough scrubbing with soap, water, and a scrub brush for 2 minutes. The current recommendation for hand washing when donning gloves is only for 15 seconds without a scrub brush.

The "glove-juice" method has been used to measure bacterial counts on the hand, a method that averages the entire hand and wrist and does not focus on the proximal phalange where rings are worn. Fagernes et al. (2007) found that total bacteria counts and Staphylococcus aureus were the same when ringed hands and non-ringed hands were compared, but wearing a single ring increased the total rate of Enterobacteria. Waterman et al. (2006) found that removing rings is recommended before surgeons perform and prepare for procedures. There were no significant differences between bacterial counts under the gloves of ring-wearers compared with non-ring-wearers. There was no increase after preparation and 3 hours of glovewearing for both hands. There was no evidence that surgeons wearing rings accumulate higher bacterial counts under their gloves during surgery.

Wongworawat and Jones (2007) looked for the effectiveness of scrubless and water-aided alcoholbased hand sanitization with a povidone-iodine scrub around finger rings. No significant difference was found in the number of bacteria comparing ringed hands and non-ringed hands that used an alcohol wash or alcohol-chlorhexidine lotion. After the povidone scrub, the number of bacteria on ringed hands was greater than unringed hands. "Use of water-less alcohol lotion resulted in the lowest bacterial count regardless of the presence of the rings."

Alp et al. (2006) found pathogenic organisms exclusively on the hands of laboratory personnel who wore jewelry (36.7\% rings, $46.9 \%$ watch, and $6.1 \%$ bracelet). Kelsall et al. (2006) found that scrubbing reduces microbes under finger rings, compared to adjacent skin or the opposite hand. By removing rings before scrubbing, the number of bacteria is reduced, but is still higher than adjacent skin or the opposite hand. These authors conclude that staff should not wear finger rings.

Trick et al. (2003) showed that wearing more rings increased the risk of contamination by organisms. Compared to plain soap and water, hand contamination with any organism was less likely after using an alcohol-based hand rub, but not after using a medicated hand wipe. Wearing rings increases the chance of hand contamination with potential nosocomial pathogens. Use of an alcohol-based hand rub resulted in less chance of hand contamination.

Montville et al. (2002) found that the presence of rings slightly reduced the efficacy of hand washing. The most important factors were sanitizer, soap, and drying method. Field et al. (1996) found that rings and watches should be removed before performing dental operative procedures. There were significantly more bacteria isolated under rings and watches. Few differences in microflora were found on the skin between surgeons and nonclinical staff, but they felt that contamination poses a threat to immunocompromised patients, especially when gloves are torn or perforated. Since disinfection under watches and rings cannot occur, these authors felt that they should be removed before disinfection and donning gloves. Kennedy et al. (2007) found that $40 \%$ of health care workers believed that rings play a role in nosocomial infections and $61 \%$ wore at least one ring to work. A disconnect existed between knowledge, beliefs and practice.

The 2002 CDC report calls for more research on this issue, which this study is designed to do.

\section{Materials and methods}

A prosthetic hand manikin made of chlorinated polyethylene elastomer (CPE) (Gettleman et al., 1987) was made from an alginate impression and plaster moulage of the prime author's left hand to fit a non-sterile medical exam glove (Powder Free 
Vinyl, No. 6370, medium, PSS World Medical, Jacksonville, USA). The glove served as a bare hand, which could be removed and discarded between tests. Metal finger rings made from mood rings with the plastic insert removed were placed over the glove on the proximal phalange of four fingers as follows: a) loose (thumb); b) snug (little finger); c) tight (middle finger); d) perforated (with a stone setting) on the ring finger; e) no ring was placed on the index finger as a control. An outer glove was not used. A controlled amount of Glo Germ $^{\mathrm{TM}}$ powder (Glo Germ ${ }^{\mathrm{TM}}$, Moab, USA) was applied with a No. 5 sable brush to the first phalange of the back and palm side of the five fingers and rings.

Digital images of the clean-gloved fingers (back and palm) were made in UV light (B-100, $100 \mathrm{~W}$ UV lamp, Ultra-Violet Products, Inc., San Gabriel, USA) before and after testing as a control. Light intensity measurements were taken using a photocell light meter (Weston Master III, Model 737, Daystrom, Inc., Weston Instruments Division, Newark, USA) at a fixed distance. The hand was then washed with a scrub brush under cold city tap water $\left(20^{\circ} \mathrm{C}\right)$ alone for 5 seconds and blotted dry with two paper towels (Bay West EcoSoft, Wausau Paper ${ }^{\mathrm{TM}}$, Harrodsburg, USA); images were made again and light meter measurements made under UV light. The gloves were changed, clean rings reapplied to the fingers, and the procedure was repeated for 15 seconds and 25 seconds washes with cold water, and then again for warm water $\left(40^{\circ} \mathrm{C}\right)$. Similar procedures were conducted with cold and warm city tap water and $1.5 \mathrm{~mL}$ of Walgreens Antibacterial Hand Soap (Walgreen Co., Deerfield, USA).

Raw light meter measurements in foot-candles were converted to lux using the formula: 1 lux (lx) $=1$ candela-steradian $/$ meter $^{2}\left(\mathrm{~cd} \cdot \mathrm{sr}^{-} \mathrm{m}^{-2}\right)=10.8$ foot-candles. The results were analyzed for the palm and the back of the hand. Delta scores were calculated as follows: light meter readings (in lux) of the fluorescence of the hand after application of Glo Germ ${ }^{\mathrm{TM}}$ minus baseline readings before application of Glo Germ ${ }^{\mathrm{TM}}$, minus readings after washing $(n=72)$ resulted in a delta final score.

A full-factorial, three-factor analysis of variance (ANOVA) was used to test for differences among levels of the three study factors-time, tempera- ture, and soap use. Tukey's post hoc honestly significant difference (HSD) test was applied to significant factors to examine pair wise differences between factor levels. All hypothesis tests were conducted at the 0.05 significance level (R, v. 2.9.1, R Foundation for Statistical Computing, Vienna, Austria).

\section{Results}

Figure 1 shows the left hand manikin with a fresh vinyl glove. Four fingers were fitted with rings [loose (thumb), snug (correct fitting on the little finger), tight (middle finger), and with a stone setting (ring finger)] or no ring control (index finger), dusted with Glo Germ ${ }^{\mathrm{TM}}$ simulated microbes, and washed with a scrub brush for 25 seconds under $20^{\circ} \mathrm{C}$ cold water without soap. The center of the image appears bright because of the intensity of the beam at the center, and artifacts of imaging in ultraviolet light with a digital camera.

Table 1 provides summary statistics for each combination of factor levels and Figure 1 provides a graphical depiction. Differences in delta scores among the three wash times were quite significant for the back of the hand $(P=0.0001)$, palm ( $P=$ $0.003)$, and for both locations combined $(P<$ $0.000,1$ ) (Table 2). For the back of the hand, delta scores with soap were significantly lower than those without soap $(P=0.03)$, a result that was not observed for the palm $(P=0.84)$ nor both locations combined $(P=0.28)$. This significant result for the back of the hand was an apparent effect of low mean delta scores for the back of the hand with cold water and soap at 5 and 15 seconds $(-0.4$ and 0.5 ). Additionally, the temperature-soap interaction term was statistically significant for the back of the hand $(P=0.002)$ as well as for both locations combined $(P=0.03)$. The phenomenon behind these significant effects can be seen in Figure 2. While the addition of soap to warm water tended to increase delta scores, the addition of soap to cold water decreased delta scores. This also was likely a byproduct of the low delta scores for the cold water with soap washes at 5 and 15 seconds.

Tukey's post hoc HSD tests showed that each pair wise comparison of wash times was strongly 

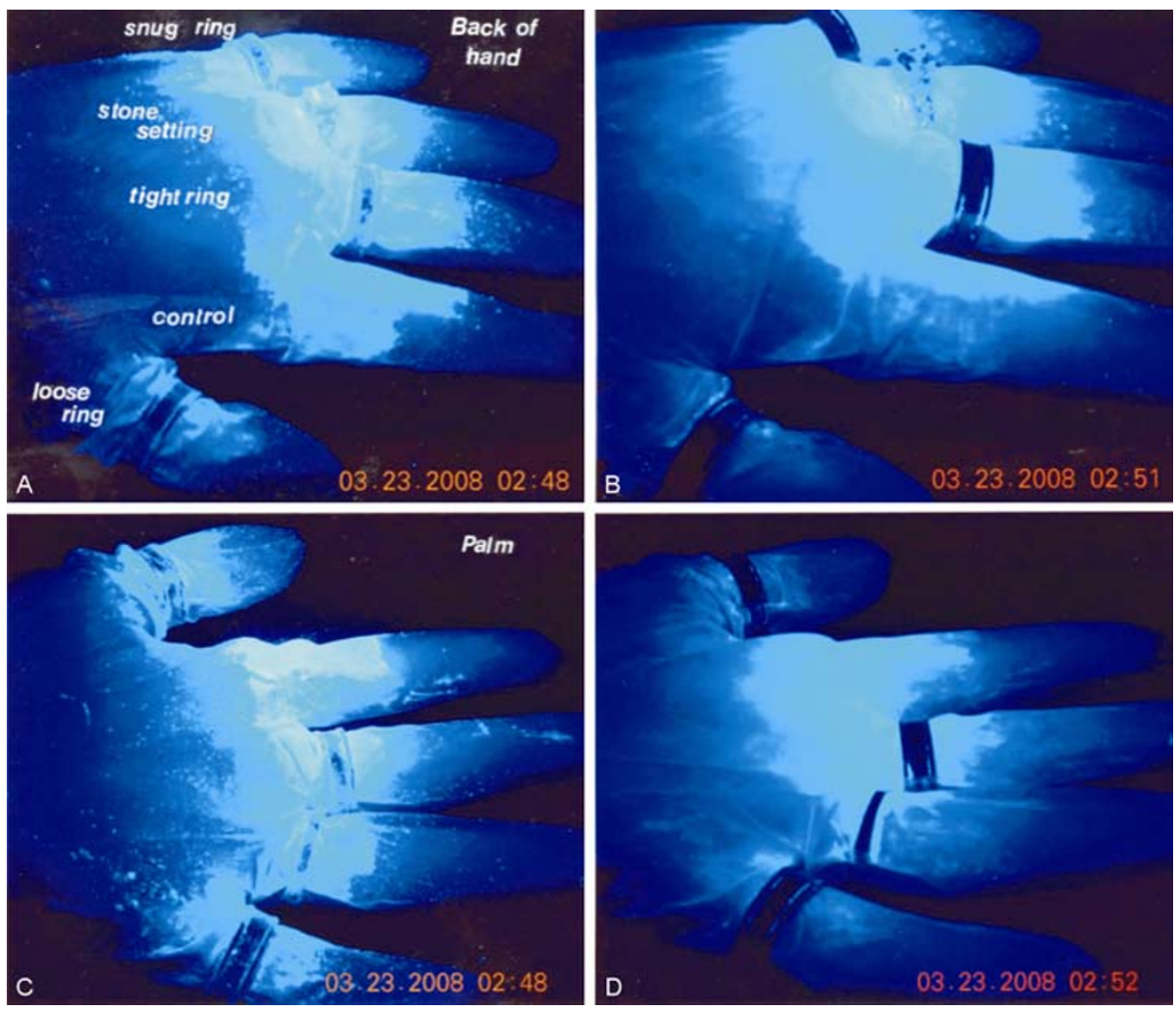

Figure 1 Light levels (in lux) of fluorescent powder before and after washing

Left hand manikin with vinyl glove. Four fingers were fitted with rings (loose-thumb, snug-little finger, tight- middle finger, and with a stone setting-ring finger) or no ring (control), dusted with Glo Germ ${ }^{\mathrm{TM}}$ simulated microbes, and washed with a scrub brush for 25 seconds under $20^{\circ} \mathrm{C}$ water without soap. The center of the image appears bright because of the intensity of the beam at the center, and artifacts of imaging in ultraviolet light with a digital camera. (A): Back of hand before washing. (B): Back of hand after washing. (C): Palm before washing. (D): Palm after washing.

Table 1 Delta score from fluorescent powder illumination

\begin{tabular}{|c|c|c|c|c|c|}
\hline Temperature & Soap & Wash time /s & Back of hand & Palm & Both \\
\hline \multirow[t]{6}{*}{ Cold } & No & 5 & $1.3(1.2)$ & $-0.2(0.1)$ & $0.6(1.1)$ \\
\hline & & 15 & $3.1(1.0)$ & $2.9(1.6)$ & $3.0(1.2)$ \\
\hline & & 25 & $5.0(0.2)$ & $4.0(0.8)$ & $4.5(0.8)$ \\
\hline & Soap & 5 & $-0.4(0.1)$ & $0.1(1.0)$ & $-0.2(0.7)$ \\
\hline & & 15 & $0.5(1.4)$ & $2.3(3.8)$ & $1.4(2.7)$ \\
\hline & & 25 & $4.6(0.8)$ & $4.3(0.6)$ & $4.5(0.6)$ \\
\hline \multirow[t]{6}{*}{ Warm } & No & 5 & $0.1(0.4)$ & $-0.7(0.2)$ & $-0.3(0.5)$ \\
\hline & & 15 & $3.5(0.4)$ & $1.3(0.8)$ & $2.4(1.4)$ \\
\hline & & 25 & $4.2(0.2)$ & $3.9(0.6)$ & $4.1(0.4)$ \\
\hline & Soap & 5 & $1.8(1.9)$ & $0.3(0.4)$ & $1.1(1.5)$ \\
\hline & & 15 & $4.3(0.3)$ & $3.6(0.7)$ & $4.0(0.6)$ \\
\hline & & 25 & $4.7(0.4)$ & $4.2(1.1)$ & $4.5(0.8)$ \\
\hline
\end{tabular}

Summary statistics for each temperature, soap, and wash time combination and each location. Values are mean (SD). $n=3$. 
Table $2 P$-values from 3-factor ANOVA models

\begin{tabular}{lclc}
\hline \multicolumn{1}{c}{ Factor } & Back of hand & Palm & Both \\
\hline Time & $0.000,1$ & 0.003 & $<0.000,1$ \\
Temperature & 0.10 & 0.60 & 0.20 \\
Soap & 0.03 & 0.84 & 0.28 \\
Time* Temperature & 0.30 & 0.60 & 0.89 \\
Time*Soap & 0.09 & 0.80 & 0.24 \\
Temperature ${ }^{*}$ Soap & 0.002 & 0.60 & 0.03 \\
Time*$^{*}$ Temperature*Soap & 0.12 & 0.39 & 0.12 \\
\hline
\end{tabular}

Table 3 Average differences (with 95\% confidence intervals) and $P$-values from Tukey's post hoc HSD test for the time factor

\begin{tabular}{|c|c|c|c|c|c|c|}
\hline \multirow[b]{2}{*}{ Comparison } & \multicolumn{2}{|c|}{ Back of Hand } & \multicolumn{2}{|c|}{ Palm } & \multicolumn{2}{|c|}{ Both } \\
\hline & Difference & $P$-value & Difference & $P$-value & Difference & $P$-value \\
\hline $15 \mathrm{~s}$ vs. $5 \mathrm{~s}$ & $2.2(1.3,3.1)$ & $<0.000,1$ & $2.7(1.3,4.0)$ & $0.000,2$ & $2.4(1.6,3.2)$ & $<0.000,1$ \\
\hline $25 \mathrm{~s}$ vs. $5 \mathrm{~s}$ & $3.9(3.0,4.8)$ & $<0.000,1$ & $4.2(2.9,5.6)$ & $<0.000,1$ & $4.1(3.3,4.9)$ & $<0.000,1$ \\
\hline $25 \mathrm{~s}$ vs. $5 \mathrm{~s}$ & $1.8(0.9,2.7)$ & $0.000,1$ & $1.6(0.2,3.0)$ & 0.02 & $1.7(0.9,2.5)$ & $<0.000,1$ \\
\hline
\end{tabular}
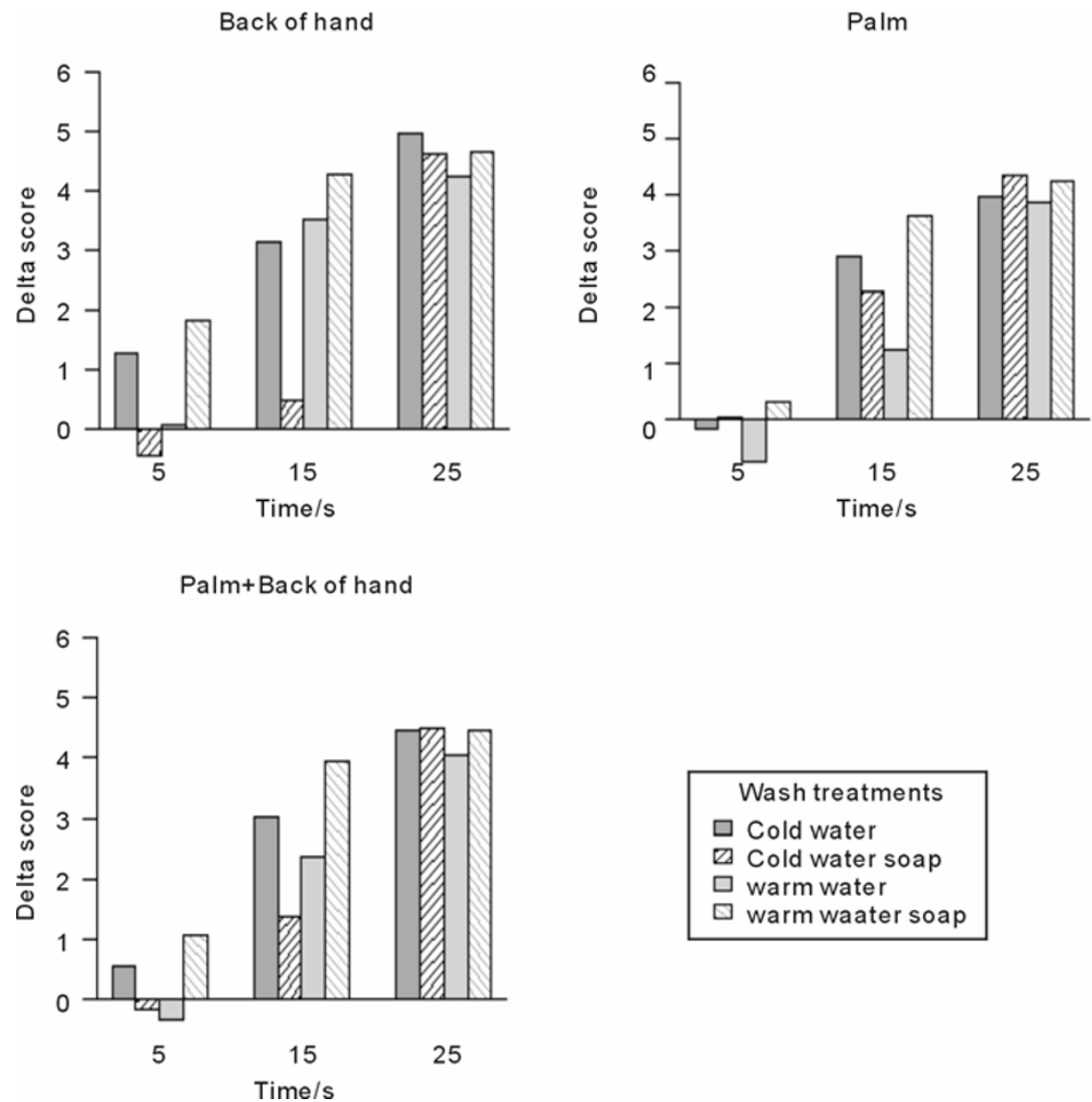

Figure 2 Bar plot of average delta scores for each temperature, soap, and wash time location

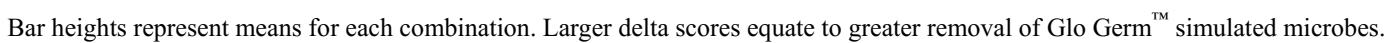


statistically significant (Table 3). Specifically, longer wash times unilaterally resulted in higher delta scores at each location.

\section{Discussion}

A model has been developed to investigate the removal of contaminants from hands wearing gloves using a hand manikin and fluorescent powder.

The overriding result of this study was that the longer the hands with rings were washed with a scrub brush under flowing water, the more simulated microbes were removed. Washing for 5 seconds was ineffective, especially on the palm. All methods were essentially equal by 25 seconds. These results should be considered preliminary due to the small numbers of replications, and the "transient" low scores for cold water + soap at 5 and 15 seconds. Cleaning the palm of the hand was less effective removing simulated microbes than the back of the hand. The liquid hand soap used in this study seemed to be more effective with warm water than cold, possibly due to the action of emulsifiers, which is more effective with higher water temperatures. Glo Germ $^{\mathrm{TM}}$ is a waxy substance that clings to surfaces, and may depart from microbial behavior in this property. Nevertheless, given a choice of cold water without soap or cold water with soap, these results indicate that the former would be preferable.

Qualitatively, the ring with the stone setting accumulated more Glo Germ ${ }^{\mathrm{TM}}$ than the other rings. After washing, the ring with the stone setting retained more simulated microbes than the other rings (see Figure 1). The upper surface of the other rings appeared clear of simulated microbes, but not the crevice beneath the ring, against the skin surface. It was not possible to distinguish small differences between the four different ring types.

If rings must be worn, it is recommended that washing and rinsing hands be extended for 25 seconds or more. CDC and WHO sources recommend only 15 seconds. It would be useful to determine the level of cross-contamination by sampling oral Streptococci that penetrate disposable examination gloves in clinical settings when wearing finger rings under the gloves.
From the results of this study, wearing rings on the fingers is not recommended because simulated microbes will remain even after 25 seconds of washing with soap and a scrub brush.

\section{Conclusions}

Under the conditions of this study, the longer the hands with rings were washed with a scrub brush under flowing water, the more simulated microbes were removed. Washing for 5 seconds was ineffective, especially on the palm. By 25 seconds, all methods were essentially the same. Washing the palm of the hand was less effective removing simulated microbes than the back of the hand. The liquid hand soap used in this study was more effective with warm water than cold. When given a choice of cold water with or without soap up to 15 seconds, these results indicate that it would be preferable not to use soap when removing simulated microbes. Qualitatively, the outer surface of finger rings were more effectively cleaned than the crevice below the ring, and the ring with a stone setting appeared to accumulate and retain simulated microbes more than other rings. Rings should not be worn under examination gloves due to difficulty cleaning in the crevices under the ring, and the consequences of cross-contamination between the patient and the health care worker.

\section{Acknowledgements}

Thanks to Mr. Robert Baar, Ballard High School, Independent Research Advisor for encouraging me to be involved in research, and my parents, Dr. Ashok Alur for supporting me immensely.

\section{References}

Alp E, Haverkate D, Voss A (2006). Hand hygiene among laboratory workers. Infect Control Hosp Epidemiol, 27(9): 978-980.

The US Centers for Disease Control \& Prevention. CDC morbidity \& mortality weekly report. Guideline for hand hygiene in health-Care settings. 51(RR16); pp.

Int J Oral Sci, 1(3): 136-142, 2009 - 141 - 
30-31. [WWW document] URL http://www.cdc.gov/ $\mathrm{mmwr} /$ preview/mmwrhtml/rr5116a1.htm [accessed October 25, 2002].

Fagernes M, Lingaas E, Bjark P (2007). Impact of a single finger ring on the bacterial load on the hands of healthcare workers. Infect Control Hosp Epidemiol, 28(10): 1191-1195.

Field EA, McGowan P, Pierce PK, Martin MV (1996). Rings and watches: should they be removed prior to operative dental procedures. J Dent, 24(1/2): 65-69.

Gettleman L, Vargo JM, Gebert PH, Rawls HR (1987). Thermoplastic chlorinated polyethylene for maxillofacial prostheses. In: Advances in Biomedical Polymers, Polymer Science and Technology Series, Vol. 35, Gebelein CG, ed. New York: Plenum, pp. 31-40.

Hoffman PN, Cooke EM, McCarville MR, Emmerson AM (1985). Micro-organisms isolated from skin under wedding rings worn by hospital staff. $\mathrm{Br}$ Med J, 290 (6463): 206-207.

Jacobson G, Thiele JE, McCune JH, Farrell LD (1985). Hand washing: ring-wearing and number of microorganisms. Nurs Res, 34(8): 186-188.

Kelsall NKR, Griggs RKL, Bowker KE, Bannister GC (2006). Should finger rings be removed prior to scrubbing for theatre. J Hosp Infect, 62(4): 450-452.

Kennedy AM, Elward AM, Fraser VJ (2004). Survey of knowledge, beliefs, and practices of neonatal intensive care unit healthcare workers regarding nosocomial infections, central venous catheter care, and hand hygiene. Infect Control Hosp Epidemiol, 25(9): 747752.

Martin MV, Dunn HM, Field EA, Field JK, Hibbert SA, McGowan $\mathrm{P}$, et al. (1988). A physical and micro- biological evaluation of the re-use of non-sterile gloves. Br Denl J, 165(9): 321-324.

McDonnell G, Haines K, Klein D, Rippon M, Walmsley R, Pretzer D (1999). Clinical correlation of a skin antisepsis model. J Microbiol Methods, 35(1): 31-35.

Montville R, Chen Y, Schaffner DW (2002). Risk assessment of hand washing efficacy using literature and experimental data. Int J Food Microbiology, 73(2/3): 305-313.

Salisbury DM, Hutfliz P, Treen LM, Bollin GE, Gautam S (1997). The effect of rings on microbial load of health care workers' hands. Am J Infection Control, 25(1): 24-27.

Trick WE, Vernon MO, Hayes RA, Nathan C, Rice TW, Peterson BJ, et al. (2003). Impact of ring wearing on hand contamination and comparison of hand hygiene agents in a hospital. Clin Infect Dis, 36(11): 13831390.

Waterman TR, Smeak DD, Kowalski J, Hade EM (2006). Comparison of bacterial counts in glove juice of surgeons wearing smooth band rings versus those without rings. Am J Infection Control, 34(7): 421425 .

Wongworawat MD, Jones SG (2007). Influence of rings on the efficacy of hand sanitization and residual bacterial contamination. Infect Control Hosp Epidemiol, 28(3): 351-353.

World Health Organization. World health organization guidelines on hand hygiene in health care. [WWW document] URL http://www. trapianti.ministerosalute. it/resources/static/primopiano/274/HH_Guidelines_10 Oct2005_AdvDraft_FINAL.PDF. [accessed October $10,2005]$.

Presented at the International Association for Dental Research $87^{\text {th }}$, Annual Meeting, Miami Beach, Florida, USA, Microbiology/Immunology Group, Infection Control Section, April 2, 2009, Poster Abstract No. 802

*Corresponding author: Lawrence Gettleman

Address: School of Dentistry, University of Louisville, 501 S. Preston St., Rm. LL 35-U, Louisville, Kentucky 40292-0001, USA

Tel: 0015028521185 Fax: $0015028527573 \quad$ E-mail: gettleman@louisville.edu 